\title{
A novel pneumatic actuator with Tensairity
}

\author{
R. H. Luchsinger ${ }^{1}$ \& M. Bräker ${ }^{1,2}$ \\ ${ }^{1}$ Empa, Center for Synergetic Structures, Switzerland \\ ${ }^{2}$ Fachhochschule Nordwestschweiz, Switzerland
}

\begin{abstract}
Inspired by pressure driven locomotion in nature, a novel pneumatic actuator concept is presented. Based on the principle of Tensairity, the actuator consists of a conical inflated hull and a compression and a tension element that are tightly connected to the hull. The flexible compression and tension elements are stabilized by internal air pressure. Acting against gravity, a first proof of concept demonstrator weighing only $300 \mathrm{~g}$ is able to lift a weight of $25 \mathrm{~N}$ a distance of almost $70 \mathrm{~cm}$ with an internal air pressure of only $20 \mathrm{kPa}$. The efficiency of the actuator is studied and optimized. Possible applications of the new actuator concept are seen in locomotion, particularly in interactions with humans or fragile objects.
\end{abstract}

Keywords: pneumatic actuator, Tensairity, efficiency.

\section{Introduction}

Pressure induced stability is a common concept in nature. As an example, the green tissue of plants is stabilized by the cellular pressure. These plants start to wilt under shortage of water, which results in a reduced cellular pressure. Next to maintaining structural integrity, pressure is also used in nature for locomotion. One interesting example is the leg of the spider. It was shown by different groups that spiders stretch their legs by pumping blood from inside their body into the cavity of the long thin leg by varying the blood pressure by up to a factor of ten [1-4]. Maximal pressure values of $60 \mathrm{kPa}$ have been observed. Some species can stretch their legs at speed rates that enable the spider to jump [3]. Inspired by this mechanism, the first pressure driven actuators were built [5].

A different approach to using pressure for actuators is inspired by the functionality of muscles. The so called McKibben artificial muscles are made of a tube like membrane that is reinforced by helically winded fibres [6]. Increasing 
the pressure in the tube leads to a thickening and accordingly to a shortening of the tube. An axial tension force results in the similar action of a muscle. Applications of the artificial, or sometimes also called fluidic, muscles are sought in prosthetics and humanoid robots. Major advantages are the light weight and compliance of these actuators. Today, they are commercially available [7].

In this paper we propose a novel pneumatic actuator. The actuator relies on the principle of Tensairity [8]. Using compliant compression and tension elements, the cone shaped actuator lifts weights along a non-linear trajectory. The interesting features of this actuator are that it is light weight and has a large load path, in combination with relatively high loads. In Section 2, a short introduction to Tensairity will be given. The design of the actuator and mode of operation will be discussed in Section 3. The experimental set up to study the efficiency of the actuator is described in Section 4. The results and discussion are given in Section 5, which is followed by the conclusions.

\section{Tensairity}

Tensairity ${ }^{\circledR}$ is a new light-weight structural concept. The synergetic combination of a pneumatic structure with cables and struts allows one to increase the load bearing capacity of conventional airbeams by order of magnitudes [8]. Outstanding properties of Tensairity are the compact transport volume, the fast and simple set up and the minimal weight in combination with the high load bearing capacity [9]. First applications of Tensairity in civil engineering, such as roof structures and bridges [10], demonstrate the potential of the technology. In the roof of a parking garage in Montreux, Switzerland, the architects made extensive use of the new formal language of the system and the interesting

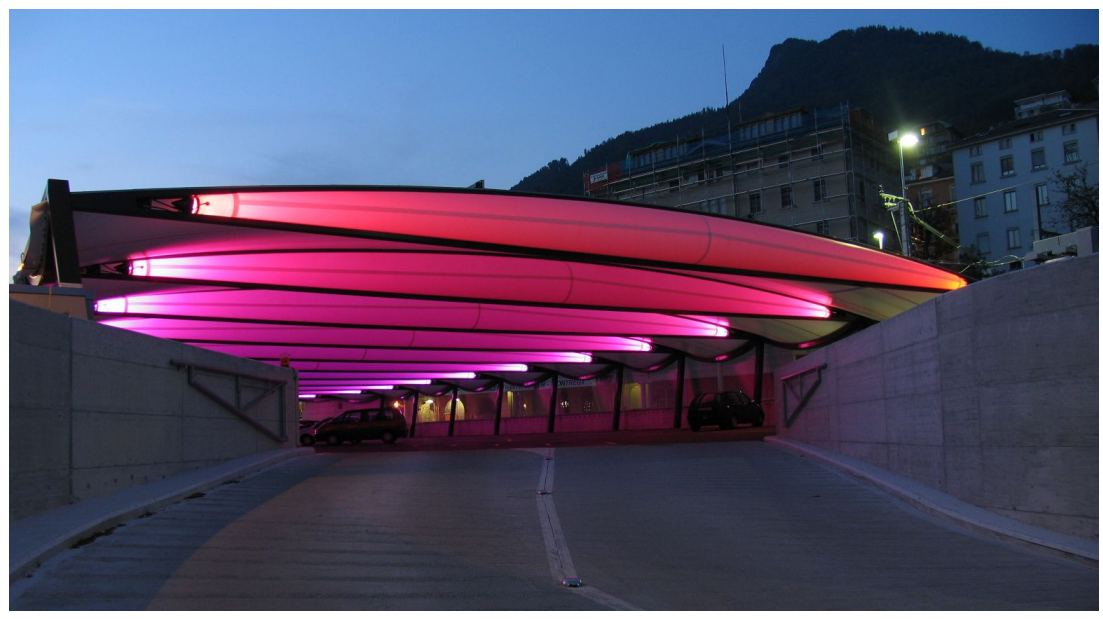

Figure 1: $\quad$ Tensairity roof structure with $28 \mathrm{~m}$ span (Airlight Ltd and Luscher Architectes, 2004). 
lighting options (fig. 1). Recently, Tensairity was applied to very light-weight wing structures [11]. The current research focuses on Tensairity columns [12] and deployable Tensairity structures [13].

\section{Tensairity actuator}

A further interesting property of Tensairity is the adaptivity. Changing the air pressure in the structure changes the stiffness. Based on this possibility, the first concept of an actuator based on Tensairity was proposed [14]. A cylindrical Tensairity structure with a flexible compression element and two helical cables was able to lift small loads when the air pressure was increased (fig. 2). The first prototype was able to demonstrate the concept of the new actuator; however, the load path was very limited and the overall behaviour was not very satisfactory. Further studies have shown that a conical shape enhances the performance of the actuator enormously. The cables were replaced by an upper tension element, which is identical to the compression element.

\subsection{Design of the Tensairity actuator demonstrator}

The Tensairity actuator consists of two flexible aluminium struts with $1.5 \times 20$ $\mathrm{mm}$ cross sectional area, which are tightly connected with pockets to the hull of a conical air chamber called the actuation chamber (black), as shown in fig. 3 . Inside the actuation chamber is a smaller air chamber called the displacement chamber (grey). This chamber is dimensioned such that it fills the volume of the actuation chamber if the actuator is fully deflected. The role of the displacement chamber is to enhance the efficiency of the actuator and to support the deformation of the struts as described in Section 3.2. The pressure of both chambers can be changed independently. For a normal actuation cycle, only the pressure of the actuation chamber is changed, while the pressure in the displacement chamber is kept constant. The hull of both chambers is made of PU coated nylon fabrics, which were welded with a high frequency welding machine. The total weight of the actuator, including the hulls and the struts, is $300 \mathrm{~g}$.
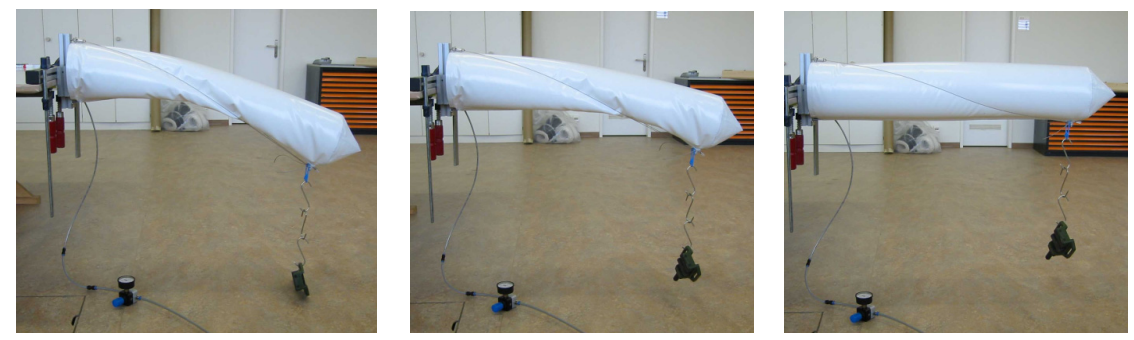

Figure 2: $\quad$ First concept of a pneumatic actuator based on Tensairity. 


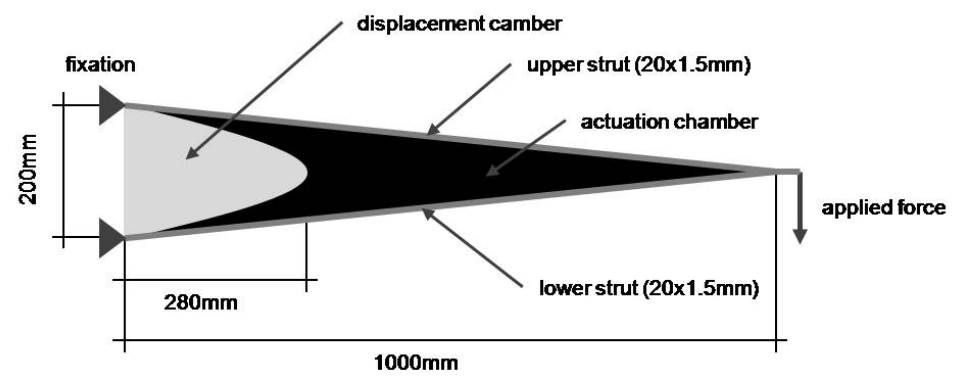

Figure 3: Dimensions of the Tensairity actuator.

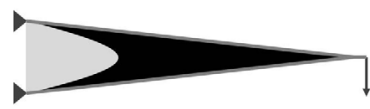

b

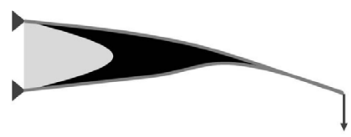

$\mathrm{C}$

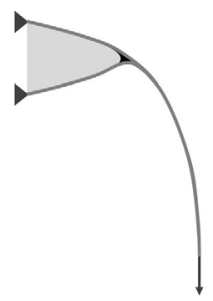

Figure 4: Schematic deformation of the struts and the change of volume of the actuation chamber under reduced air pressure.

The volumes of the chambers were determined by measuring the air volume needed to fill the empty chamber by using a flow meter. The displacement chamber has a volume of 5.0 litres, whereas the total volume of the actuator is 13.1 litres. Depending on the pressure in the displacement chamber, the volume of the actuation chamber can be varied between 8.1 litres to 13.1 litres.

\subsection{Working principle of the Tensairity actuator}

The pressure in the actuation chamber is separating and stabilizing the upper and the lower strut of the actuator. In the fully stretched position with maximal pressure, the actuator is identical to a conventional Tensairity cantilever (fig. 4a). The upper strut is under tension, while the lower strut is under compression. The pressurized hull of the actuation chamber stabilizes the lower strut against buckling. This is the basic concept of Tensairity. If the pressure of the actuation chamber is reduced, the stabilization of the compression strut decreases and the strut starts to buckle (fig. 4b). It is very important that this buckling starts at the tip of the actuator and that the deformation gradually increases and shifts towards the base of the actuator. In this way, a continuous reversible movement of the load can be achieved. For low pressure values, the displacement chamber helps to stabilize the compression strut at the base (fig. 4c). Thus, the displacement chamber also has a supporting function. 

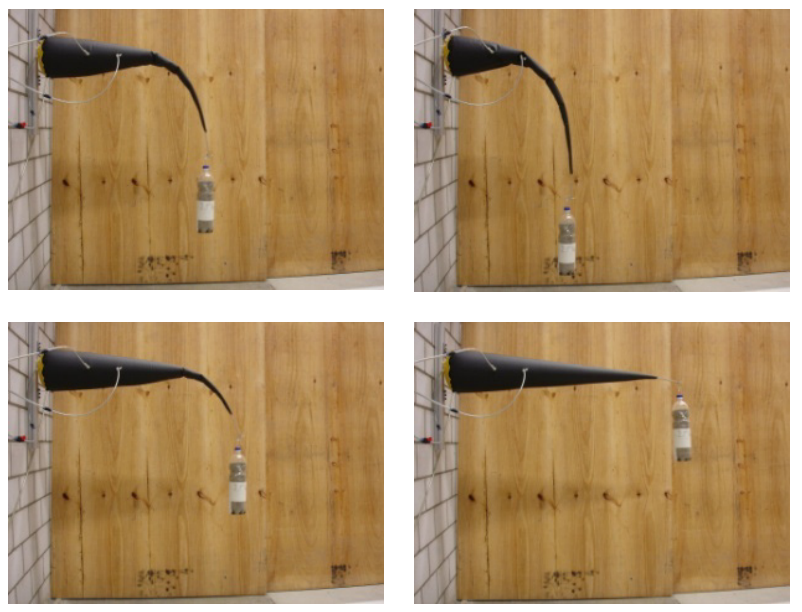

Figure 5: Lift process of the Tensairity actuator.

Four steps of the work cycle of the Tensairity actuator demonstrator are shown in fig. 5. In the initial configuration, the pressure of the actuation chamber is identical to the ambient pressure and the volume of the actuation chamber is almost identical to the volume of the displacement chamber. The lower strut is stabilized at the base by the displacement chamber. Increasing the pressure in the actuation chamber slowly straightens the compression element from the base towards the tip leading to a rolling lift of the load similar to the movement of a trunk of an elephant. In the final configuration the actuator is completely stretched.

\section{Experimental setup}

In order to investigate the efficiency of the novel pneumatic actuator, the set up as shown in fig. 5 is used. A specific weight is attached to the tip of the actuator at the initial configuration. The pressure in the displacement chamber $p_{d}$ is measured and kept constant during the load cycle. Pressurized air is released at constant initial pressure $p_{\text {in }}$ into the actuation chamber to start the lifting during which the pressure in the actuation chamber $p_{a}$ increases. The pressure in the actuation chamber is measured as well as the flow of air into the chamber. The vertical displacement of the load is recorded by means of an ultrasonic distance sensor.

The efficiency of the actuator $\eta$ is defined as the work done by the actuator divided by the compression energy used to transfer the air from environmental conditions into the state inside the actuation chamber. Several assumptions were made:

- Stable environmental conditions ( $T_{0}=$ const. and $p_{0}=$ const. $)$.

- Isothermal compression during lifting process $\left(T=T_{0}=\right.$ const. $)$.

- Ideal gas theory. 
- The volume of the fully filled actuator is not dependant on the pressure inside the chambers.

The work is given by

$$
W=m_{l} \cdot g \cdot h
$$

with $m_{l}$ the mass of the load, $g$ the free-fall acceleration, $h$ the vertical displacement of the load. Under the given assumptions the compression energy of the air in the actuation chamber is

$$
U=m_{a} \cdot R_{\text {air }} \cdot T_{0} \cdot \ln \frac{p_{a}}{p_{0}}
$$

with $m_{a}$ the mass of the air in the actuation chamber, $p_{a}$ the pressure of the air in the actuation chamber, $p_{0}$ and $T_{0}$ the ambient pressure and temperature and $R_{\text {air }}=287 \mathrm{~J} / \mathrm{kg} / \mathrm{K}$ the gas constant of air. The efficiency of the actuator is then given by

$$
\eta(t)=\frac{m_{l} \cdot g \cdot h(t)}{m_{a}(t) \cdot R_{\text {air }} \cdot T_{0} \cdot \ln \frac{p_{a}(t)}{p_{0}}} .
$$

The measured mass flow of the air into to actuation chamber $\dot{m}_{a}(t)$ needs to be integrated over time to obtain the mass of the air in the chamber

$$
m_{a}(t)=m_{a}(0)+\int_{0}^{t} \dot{m}_{a} d t .
$$

The mass of the air in the actuation chamber in the final straight position is given by the pressure and the given maximal volume of the actuation chamber (Sec. 3.1). The initial mass $m_{a}(0)$ can thus be determined by subtracting the measured air mass which was flown into the actuator during the load lifting from the total mass at the end of the lifting. Together with the measured quantities $p_{a}(\mathrm{t})$ and $h(\mathrm{t})$ the efficiency can be evaluated.

\section{Results and discussion}

The influence of various parameters on the behaviour of the Tensairity actuator was investigated in detail. The role of the pressure in the displacement chamber on the compression energy of the air is shown in fig. 6. As can be seen, the higher the pressure in the displacement chamber, the smaller the volume to be filled in the actuation chamber and thus less compression energy is needed. This, of course, also increases the efficiency of the actuator, which is a major reason for using the displacement chamber. Based on this result, we use $p_{d} \geq p_{a}$ in all the following experiments.

In fig. 7 on the left, the flow of the air mass into the actuation chamber is shown for different values of the inlet pressure $p_{i n}$. As expected, the air flow increases with $p_{i n}$ and thus the time to lift the load decreases. The efficiency is mainly shifted in time for different values of $p_{\text {in }}$ (fig. 7, right). The maximal values are almost identical. 


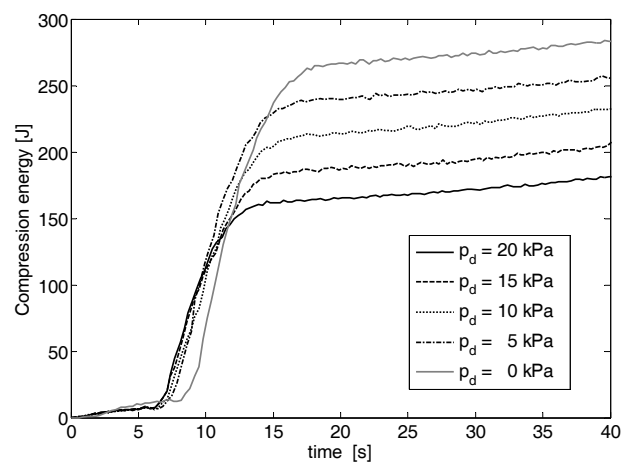

Figure 6: Compression energy of the air during the lifting for different pressures in the displacement chamber $\left(m_{l}=1 \mathrm{~kg}, p_{\text {in }}=20 \mathrm{kPa}\right)$.
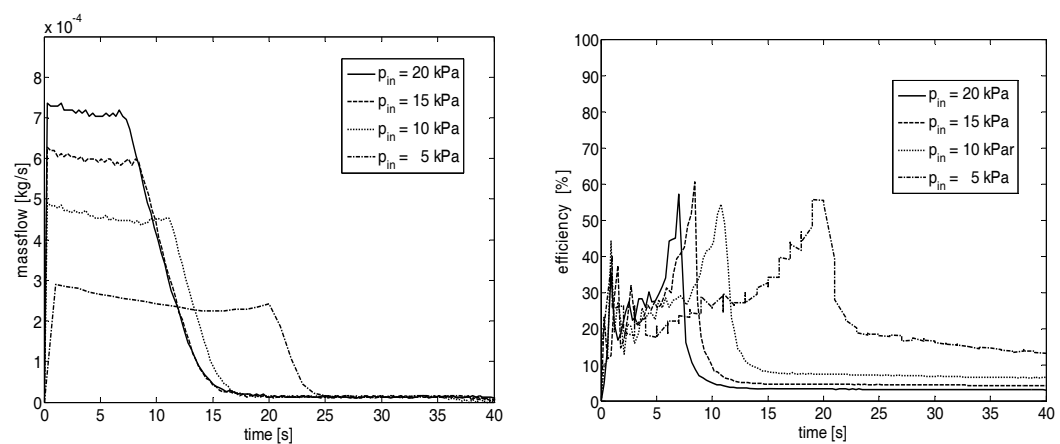

Figure 7: Flow of air and efficiency for different inlet pressure values $\left(m_{l}=1 \mathrm{~kg}, p_{d}=20 \mathrm{kPa}\right)$.

The behaviour of the actuator depends on the load to be lifted. In fig. 8 on the left, the height of the load as a function of the pressure in the actuation chamber is shown for different values of the attached mass. A higher mass leads to a higher deflection of the actuator at the initial configuration with the pressure in the actuation chamber equal to the ambient pressure. Furthermore, to reach the same height a higher pressure in the actuation chamber is needed.

The work done by the actuator for different load values is shown in fig. 8 on the right. As the work is proportional to the mass of the load and the maximum displacement increases with load, higher masses must lead to a higher work done by the actuator. If the weight is doubled, the work is approximately 2.5 times higher. From this graph it also becomes obvious that the lifting lasts for about 7 seconds for the $1 \mathrm{~kg}$ load while it takes about 12 for the $2.5 \mathrm{~kg}$ load. After that, the actuator remains straight and the work remains constant. 

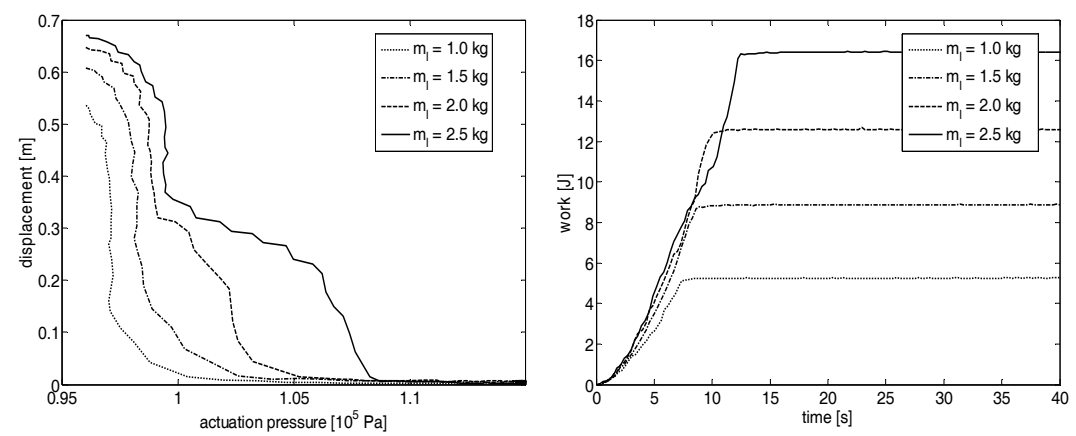

Figure 8: The influence of the load on the behaviour of the actuator. Deflection as a function of $p_{a}$ (left) and works as a function of time (right) $\left(p_{\text {in }}=p_{d}=20 \mathrm{kPa}\right)$.
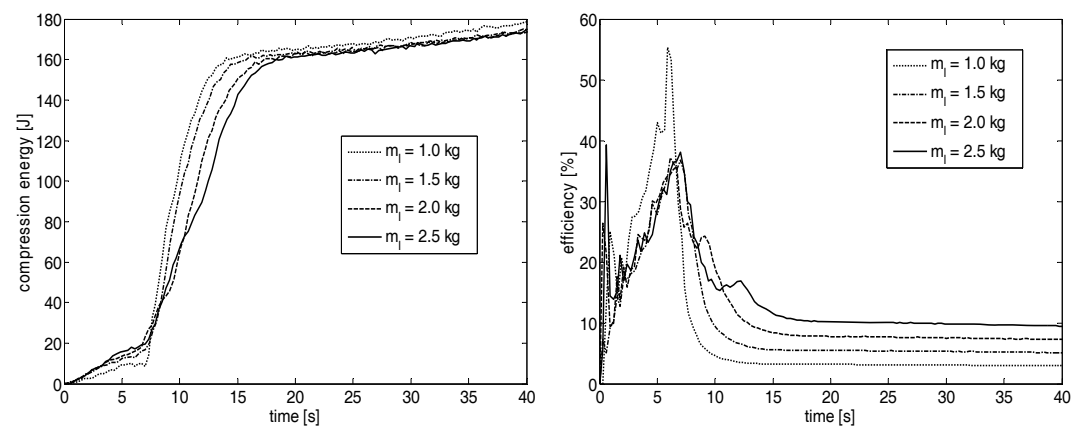

Figure 9: The influence of the load on the compression energy (left) and the efficiency (right) as a function of time $\left(p_{i n}=p_{d}=20 \mathrm{kPa}\right)$.

The compression energy of the air in the actuation chamber is shown in fig. 9 on the left for different loads. In the work phase up to about 7 to 12 seconds (fig. 8 , right), the compression energy is smaller for smaller mass. The reason for this is that less pressure is needed to lift smaller loads as shown in fig. 8, left. In the second phase up to a time of about fifteen seconds, the actuator is already almost straight and the volume of the actuation chamber is more or less constant. However, the compression energy further increases since $p_{a}$ increases to the value of $p_{\text {in }}$ until equilibrium is reached. Thus, the compression energy still increases during this phase; however, no work is done anymore. The total compression energy is independent of the load since the final pressure and the volume of the actuation chamber is identical for all four load values.

The efficiency of the actuator is shown in fig. 9 at the right. With a load of 1 $\mathrm{kg}$ a maximum of $55 \%$ close to the end of the lifting is achieved. The other loads have smaller but similar maximum efficiency values somehow below $40 \%$. The higher efficiency for the $1 \mathrm{~kg}$ load can be explained by the fact that only for this 
load a completely straight compression strut is obtained by a sudden snap to this position at the tip. From this point on, the geometry of the actuator will not change further with increasing pressure. This is also reflected in fig. 9, left in the sudden kink of the compression energy for the load of $1 \mathrm{~kg}$ at about seven seconds. For all higher loads, the compression strut only gradually approaches the straight position. Therefore, the transition in the compression energy around 7 seconds is less pronounced.

The efficiency as a function of the displacement is shown in fig. 10 for the four load values. The actuator moves the biggest part of its actuation path at a small pressure (fig. 8, left). This is the operation range of the actuator with high efficiency. For the last few millimetres of the movement the pressure in the actuation chamber rises, however, not much work is done anymore. Thus, one can define a work region for the actuator with reasonable high efficiency. For a load of $2 \mathrm{~kg}$ one might define this region to be between horizontal displacements of $-0.5 \mathrm{~m}$ and $-0.05 \mathrm{~m}$, where the efficiency ranges between $20 \%$ and $35 \%$.

\section{Conclusions}

A novel pneumatic actuator based on the concept of Tensairity is presented. The simple set up mainly consist of a conical inflatable hull and two flexible struts. An internal displacement chamber enhances the stabilisation at low pressure values in the actuation chamber and increases the efficiency of the actuator. Investigations have shown that the pressure of the displacement chamber should be higher than the pressure in the actuation chamber. The attached load influences the work done as well as the compression energy of the air. Therefore, a higher efficiency results for smaller loads. The average efficiency of the actuator in a specified working range is about $30 \%$. Combining low weight, large working path with reasonable loads, possible applications of the new actuator concept are seen in locomotion, especially in interactions with humans or fragile objects.

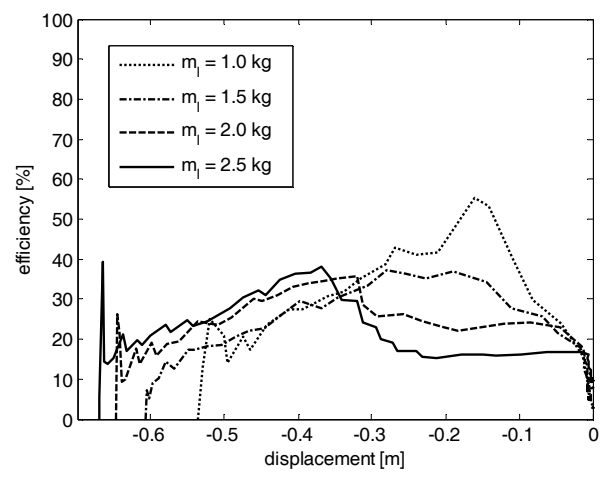

Figure 10: The efficiency of the actuator as a function of the horizontal displacement for different loads $\left(p_{i n}=p_{d}=20 \mathrm{kPa}\right)$. 


\section{Acknowledgements}

The authors would like to thank L. Düring and M. Senteler for their contributions in the development of the actuator. The financial support of Festo is gratefully acknowledged.

\section{References}

[1] Ellis, C.H., The mechanism of extension in the legs of spiders. The biological bulletin, 86, pp. 41-50, 1944.

[2] Perry, D.A. \& Brown, R.H.J., The hydraulic mechanism of the spider leg. Journal of experimental biology, 36, pp. 423-433, 1959.

[3] Perry, D.A. \& Brown, R.H.J., The jumping mechanism of salticid spiders. Journal of experimental biology, 36, pp. 654-665, 1959.

[4] Wilson, R.S., Some comments on the hydrostatic system of spiders (Chelicerata, Araneae). Zoomorphology, 68, pp. 308-322, 1970.

[5] Menon, C. \& Lira, C., Active articulation for future space applications inspired by the hydraulic system of spiders. Bioinspiration and biomimetics, 1-2, pp. 52-61, 2006.

[6] Chou, C.P. \& Hannaford, B., Measurements and modelling of McKibben pneumatic artificial muscles. Transaction on robotics and automation, 12(1), pp. 90-102, 1966.

[7] Festo, www.festo.com, fluidic muscle.

[8] Luchsinger, R.H., Pedretti, A., Steingruber, P. \& Pedretti, M., The new structural concept Tensairity: Basic principles. Progress in structural engineering, mechanics and computation, ed. A. Zingoni, Balkema, The Netherlands, 2004.

[9] Luchsinger, R.H., Crettol, R. \& Plagianakos, T. S., Temporary structures with Tensairity. International Symposium IASS-SLTE 2008, 3rd Latin American Symposium on Tensile-Structures, Acapulco, Mexico, 2008.

[10] Luchsinger, R.H., Crettol, R. \& Plagianakos, T., The new lightweight structure Tensairity. Proceedings of the Structural Engineers World Congress 2007, Bangalore, India, 2007.

[11] Breuer, J., Ockels, W. \& Luchsinger, R.H., An inflatable wing using the principle of Tensairity. Proceedings of the $48^{\text {th }}$ AIAA/ASME/ ASCE/AHS/ASC structures, structural dynamics and materials conference, Hawaii, 2007.

[12] Plagianakos, T.S., Teutsch, U., Crettol R. \& Luchsinger, R.H., Static response of a spindle-shaped Tensairity column to axial compression. Engineering Structures 31 (8), pp. 1822-1831, 2009.

[13] De Laet, L., Luchsinger, R.H., Crettol, R., Mollaert, M. \& De Temmermann, N., Deployable Tensairity Structures. Journal of the International Association for Shell and Spatial Structures 50 (2), pp. 121128, 2009.

[14] Luchsinger, R.H., \& Crettol, R., Adaptable Tensairity. Proceedings of the joint CIB, Tensinet, IASS International Conference on Adaptability in Design and Construction, Vol. 2, 3-7, Eindhoven, 2006. 